\title{
Extent of resection of peritumoral diffusion tensor imaging-detected abnormality as a predictor of survival in adult glioblastoma patients
}

\author{
Jiun-Lin Yan, MD, ${ }^{1,2,4,5}$ Anouk van der Hoorn, MD, PhD, ${ }^{1,3,6}$ Timothy J. Larkin, PhD,1 \\ Natalie R. Boonzaier, MSc, ${ }^{1}$ Tomasz Matys, PhD, ${ }^{3}$ and Stephen J. Price, PhD, FRCS ${ }^{1}$ \\ ${ }^{1}$ Cambridge Brain Tumor Imaging Laboratory, Division of Neurosurgery, ${ }^{2}$ Wolfson Brain Imaging Centre, Department of Clinical \\ Neuroscience, and 'Department of Radiology, University of Cambridge, Addenbrooke's Hospital, Cambridge, United Kingdom; \\ ${ }^{4}$ Department of Neurosurgery, Chang Gung Memorial Hospital, Keelung; ${ }^{5}$ Chang Gung University College of Medicine, Taoyuan, \\ Taiwan; and ${ }^{6}$ Department of Radiology, University Medical Centre Groningen, University of Groningen, The Netherlands
}

OBJECTIVE Diffusion tensor imaging (DTI) has been shown to detect tumor invasion in glioblastoma patients and has been applied in surgical planning. However, the clinical value of the extent of resection based on DTI is unclear. Therefore, the correlation between the extent of resection of DTI abnormalities and patients' outcome was retrospectively reviewed.

METHODS A review was conducted of 31 patients with newly diagnosed supratentorial glioblastoma who underwent standard 5-aminolevulinic acid-aided surgery with the aim of maximal resection of the enhancing tumor component. All patients underwent presurgical MRI, including volumetric postcontrast T1-weighted imaging, DTI, and FLAIR. Postsurgical anatomical MR images were obtained within 72 hours of resection. The diffusion tensor was split into an isotropic (p) and anisotropic (q) component. The extent of resection was measured for the abnormal area on the p, q, FLAIR, and postcontrast T1-weighted images. Data were analyzed in relation to patients' outcome using univariate and multivariate Cox regression models controlling for possible confounding factors including age, $0^{6}$-methylguanine-DNA-methyltransferase methylation status, and isocitrate dehydrogenase-1 mutation.

RESULTS Complete resection of the enhanced tumor shown on the postcontrast T1-weighted images was achieved in 24 of 31 patients (77\%). The mean extent of resection of the abnormal p, q, and FLAIR areas was 57\%, 83\%, and $59 \%$, respectively. Increased resection of the abnormal $p$ and $q$ areas correlated positively with progression-free survival $(p=$ 0.009 and $p=0.006$, respectively). Additionally, a larger, residual, abnormal q volume predicted significantly shorter time to progression $(p=0.008)$. More extensive resection of the abnormal $q$ and contrast-enhanced area improved overall survival ( $p=0.041$ and 0.050 , respectively).

CONCLUSIONS Longer progression-free survival and overall survival were seen in glioblastoma patients in whom more DTI-documented abnormality was resected, which was previously shown to represent infiltrative tumor. This highlights the potential usefulness and the importance of an extended resection based on DTI-derived maps.

http://thejns.org/doi/abs/10.3171/2016.1.JNS152153

KEY WORDS glioblastoma; extent of resection; diffusion tensor imaging; volumetric study; progression-free survival; prognosis; oncology

$\mathrm{G}$ LIOBLASTOMA is the most prevalent malignant primary brain tumor and one of the leading cancers in terms of years of life lost. ${ }^{15}$ Standard treatment for this highly malignant disease includes maximal safe re- section followed by concomitant chemoradiotherapy and adjuvant chemotherapy with temozolomide. However, the prognosis remains poor, with 2- and 5-year survival rates of only $27 \%$ and $9.8 \%$, respectively. ${ }^{27}$

ABBREVIATIONS 5-Ala = 5-aminolevulinic acid; DT = diffusion tensor; DTI = diffusion tensor imaging; EOR = extent of resection; FLAIR = fluid-attenuated inversion recovery; FLIRT = FMRIB Linear Image Registration Tool; FMRIB = Oxford Centre for Functional MRI of the Brain; FOV = field of view; FSL = FMRIB Software Library; IDH-1 = isocitrate dehydrogenase-1; HR = hazard ratio; MGMT = $0^{6}$-methylguanin-DNA-methyltransferase; $p=$ isotropic component; $q=$ anisotropic component.

SUBMITTED September 16, 2015. ACCEPTED January 12, 2016.

INCLUDE WHEN CITING Published online April 8, 2016; DOI: 10.3171/2016.1.JNS152153. 
The main factors influencing prognosis are age, performance status, tumor molecular type, and extent of resection (EOR), of which the former three are fixed and the latter can be changed. Therefore, many efforts have made to improve the extent of maximal tumor resection while preserving normal brain tissue and function. Resection of a larger fraction of the tumor results in a longer life expectancy. ${ }^{6,7,12}$ To achieve maximal tumor resection, intraoperative neuronavigation, 5-aminolevulinic acid (5ALA) and other imaging techniques (e.g., intraoperative MRI or ultrasound) have been introduced. Use of 5-ALA increased the EOR to $65 \%$ of the total contrast-enhanced area and prolong the progression-free survival. ${ }^{24} \mathrm{~A}$ contrast-enhanced T1-weighted signal has many limitations in accuracy of the delineation of tumor margin..$^{19}$ Consequently, the clinical benefit of resection outside the contrast-enhanced area has been investigated..$^{13}$ Recently, it has been shown that extending the resection to the peritumoral high T2 signal areas beyond the enhanced lesion on postcontrast T1-weighted images can provide longer survival than less extensive resections. ${ }^{13}$ This is thought to be due to tumor infiltration beyond the contrast-enhanced area. ${ }^{20}$ However, a high signal on T2-weighted imaging is not specific for tumor infiltration, because it is also caused by edema.

Diffusion tensor imaging (DTI) is able to detect tumor extent beyond the contrast-enhanced area because of subtle white matter changes. ${ }^{17}$ By decomposing the diffusion tensor into an isotropic component (p) and an anisotropic component (q) ${ }^{16}$ white matter that is infiltrated or disrupted by the glioblastoma can be identified. ${ }^{17}$ Radiotherapy treatment plans based on peritumoral abnormal DTI areas were able to achieve a large reduction of $15 \%-35 \%$ in planning target dose and $50 \%$ of clinical target volume, which minimized radiation complication without affecting the survival. ${ }^{1,8}$ In addition, DTI changes, especially those determined by p mapping, can be used to evaluate early treatment response after temozolomide chemotherapy in glioma patients. ${ }^{2}$ However, to our knowledge, there are no studies to date showing a clinical benefit of resecting the abnormal peritumoral DTI area. This prompted our study analyzing the influences of the EOR of abnormal peritumoral DTI areas on patients' outcome.

\section{Methods \\ Patient Population}

We included 31 glioblastoma patients (mean age 56 years, range 31-68 years; 19 men and 12 women) from our consecutive cohort. All patients had a Karnofsky Performance Scale score of 70 or higher and had presurgical MR scans with follow-up MRI available after tumor resection. Patients were deemed suitable for undergoing complete resection of the contrast-enhancing tumor by 1 neurosurgeon (S.J.P.), and, with the goal of achieving maximum tumor resection, tumor resection was performed using neuronavigation (StealthStation; Medtronic) and 5-ALA fluorescence guidance. The presurgical images were acquired, on average, 1 day (range 0-9 days) before surgery. Follow-up MR scans were obtained as soon as possible after surgery and no later than 72 hours after surgery. Ex- clusion criteria were previous cranial surgery, previous cerebral radiotherapy, or a known other primary tumor.

All patients received standard concomitant and adjuvant temozolomide chemoradiotherapy ${ }^{27}$ about 1 month after resection. $\mathrm{O}^{6}$-methylguanin-DNA-methyltransferase (MGMT) methylation was determined by pyrosequencing of the DMR2 region. Isocitrate dehydrogenase (IDH-1) $\mathrm{R} 132 \mathrm{H}$ mutation status was analyzed using immunohistochemistry. Survival data were extracted from the medical records. Tumor eloquence was classified as previously described..$^{12,22}$ The study was approved by the local institutional review board, and informed written consent was obtained from all patients.

\section{MRI Data Acquisition}

Presurgical imaging was performed using a 3.0-T MR Magnetom system (Siemens Healthcare) with a standard 12-channel head coil. Imaging included T1-weighted imaging after contrast, T2-weighted FLAIR sequence, and DTI. A 3D T1-weighted scan with fat suppression was acquired after the intravenous injection of $9 \mathrm{ml}$ of gadolinium (Gadovist; Bayer Schering Pharma) (TR/TE/TI 2300/2.98/900 msec; flip angle $9^{\circ}$; FOV $256 \times 240$ mm; 176-192 slices; no slice gap; and voxel size $1 \times 1 \times 1 \mathrm{~mm})$. A 2D FLAIR sequence was obtained (TR/TE/TI 7840-8420/95/2500 msec; flip angle $150^{\circ}$; FOV $250 \times 200 \mathrm{~mm}$; 25-27 slices; 1 -mm slice gap; and voxel size $0.78125 \times 0.78125 \times 4 \mathrm{~mm}$ ). DTI data were acquired using a single-shot echo-planar sequence (TR/TE 8300/98 msec; flip angle 90 FOV $192 \times$ $192 \mathrm{~mm}$; 63 slices; no slice gap; and voxel size $2 \times 2 \times 2$ $\mathrm{mm})$ with multiple b-values $(0,350,650,1000,1300$, and $1600 \mathrm{sec} / \mathrm{mm}^{2}$ ) scanned in 13 directions.

Direct postsurgical images were acquired on a $1.5-\mathrm{T}$ Optima, a 1.5- or 3.0-T Signa, or a 3.0-T Discovery MR scanner (GE Healthcare) with standard head coil. Imaging included a T1-weighted anatomical sequence after the intravenous injection of $9 \mathrm{ml}$ of gadolinium. This was performed as a 2D T1-weighted sequence (TR/TE 460-700/11-21 msec, flip angle $90^{\circ}$; FOV 220-260 × 220-260 mm; 20-85 slices; 0- to 1-mm slice gap; voxel size $0.429-0.5079 \times 0.429-0.5079 \times 2-6 \mathrm{~mm})$ or a $2 \mathrm{D} \mathrm{T} 1$ inversion recovery sequence (TR/TE/TI 2508-2600/1242/780-920 msec; flip angle 90-110 ; FOV $220 \times 220$ $\mathrm{mm}$; 20-22 slices; 1 - to 3.5-mm slice gap; and voxel size $0.4297 \times 0.4297 \times 6 \mathrm{~mm}$ ).

\section{Image Processing}

DT images were processed using tools from the Oxford Centre for Functional MRI of the Brain (FMRIB) Software Library (FSL) version 5.0.0 (fsl.fmrib.ox.ac.uk/ fsl/fslwiki). DT images were realigned to the b0-image to compensate for eddy currents and motion..$^{10}$ We calculated the isotropic component (p) and anisotropic component (q) after eigenvalues were calculated on multiple fiber directions at each voxel in the DTI data, as previously described.$^{16}$ For each subject, presurgical DT and FLAIR images were coregistered with presurgical postcontrast T1-weighted images by a linear transformation, using the default FMRIB linear image registration tool (FLIRT) functions provided by FSL. ${ }^{0}$ 
Coregistration between postsurgical and presurgical images was done by a semiautomatic coregistration methods. All images were fit into presurgical, volumetric contrast-enhanced T1-weighted images. First, we used automatic brain-extracted images, ${ }^{9}$ which were manually corrected. Then we calculated the transformation between presurgical tumor and postsurgical resection cavity by using the linear FLIRT coregistration of the lesion, ventricle, and the external mask of the brain between presurgical and postsurgical images. This transformation was then used as input transformation matrix for an FMRIB nonlinear image registration tool to coregister the brain.

\section{Tumor Volume and Extent of Resection Data Analysis}

Extent of resection was determined from the resection cavity in the coregistered, postsurgical, postcontrast T1weighted image (Fig. 1, blue outline) by one of the authors (J.L.Y.) blinded to the outcome, with the agreement of a second author (A.H.). The 3D, peritumoral, abnormal FLAIR, $p$, and $q$ regions of interest were manually selected on the coregistered presurgical MR images (Fig. 1B-1D). The interobserver correlation was done by the first author (J.L.Y.) and senior author (S.J.P.) with fair agreement. The coregistered resected region was extracted from the total abnormal presurgical region for each sequence, using Matlab (MathWorks Inc.). The resected volumes were calculated for the $\mathrm{p}, \mathrm{q}$, and FLAIR regions by multiplying all voxels of interests with the slice thickness in Matlab.

\section{Statistical Analysis}

Data were analyzed using SPSS version 22 (IBM Inc.). A Cox regression model was used to estimate the influence of the EOR based on p, q, FLAIR, and postcontrast T1weighted images on progression-free survival and overall survival. Multivariate analysis included the following covariates: age, MGMT methylation status, IDH-1 mutation status, presurgical tumor volume based on postcontrast T1-weighted images, tumor location based on eloquence, and midline shift. These covariates were tested individually with the resection ratio of $p, q$, FLAIR, enhancing area on postcontrast T1-weighted image, and residual tumor volume based on different MR images. Subgroup analysis was done for a group with low and high abnormal $q$ resection ratios by categorizing the patient based on the median of the whole group. Comparison of characteristics of patients in different groups was performed by using a ttest for continuous data because they showed a normal distribution tested by D'Agostino-Pearson normality test and the chi-square for dichotomous data. The chi-square test with Yates's correction was used for small-number contingency categorical analysis. A two-sided $\mathrm{p}$ value of 0.05 was used throughout. Mean results are presented \pm SD.

\section{Results}

\section{Patients' Characteristics}

Patients' clinical characteristics are shown in Table 1. Complete resection based on postcontrast T1-weighted imaging was achieved in 24 patients (77\% of 31 patients). Ten patients had the tumor located within an eloquent area, which included the primary motor or sensory cortex, speech center, internal capsule, and basal ganglia. Ten patients had tumor located in a near-eloquent area, which included the supplementary motor area, corpus callosum, and proximity to the calcarine fissure and the speech center. The mean midline shift was $3.3 \mathrm{~mm}( \pm 3.7$ $\mathrm{mm}$; range $0-11.9 \mathrm{~mm})$. The mean presurgical, contrastenhanced tumor volume was $46 \mathrm{ml}( \pm 30 \mathrm{ml}$; range $8-119$ $\mathrm{ml})$. The volume of the resection area was $53 \mathrm{ml}( \pm 31$
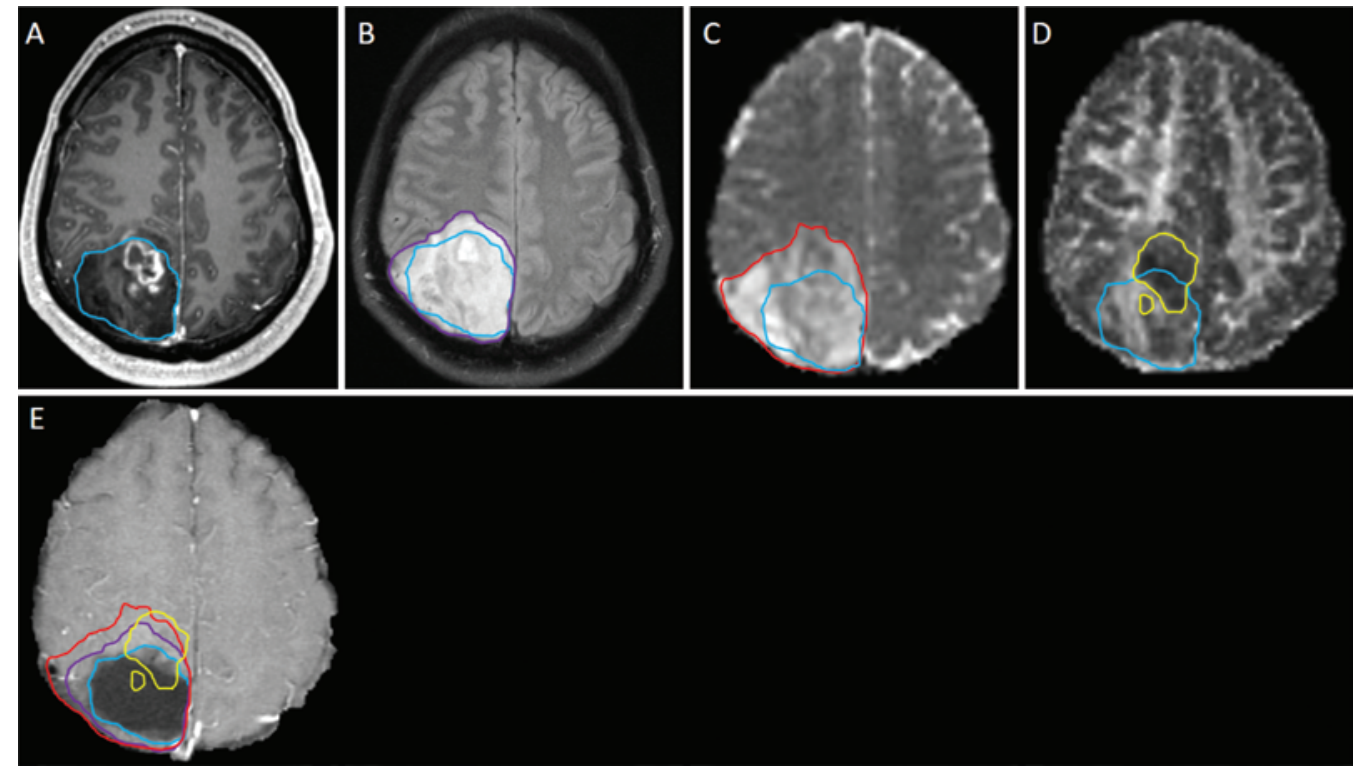

FIG. 1. Regions of interest and EOR. Presurgical postcontrast T1-weighted image (A), FLAIR (B), and diffusion tensor images (C and $\mathbf{D})$ are shown with the resected area contoured in blue in a representative patient. The abnormal FLAIR region (B, purple), the isotropic abnormality $(\mathrm{C}, \mathrm{red})$ and the anisotropic abnormality (C, yellow) are outlined. $\mathrm{E}$ : Summary of the regions of interest in a postsurgical contrast-enhanced T1-weighted image. 
TABLE 1. Patient characteristics*

\begin{tabular}{|c|c|}
\hline Characteristic & Value \\
\hline Total no. of patients & 31 \\
\hline Male & 19 \\
\hline Female & 12 \\
\hline Age, yrs & $56 \pm 11$ \\
\hline \multicolumn{2}{|l|}{ Tumor location, no. } \\
\hline Eloquent & 10 \\
\hline Near eloquent & 12 \\
\hline Noneloquent & 9 \\
\hline Midline shift, mm & $3.3 \pm 3.7$ \\
\hline \multicolumn{2}{|l|}{ Preop tumor size, by imaging type, ml } \\
\hline $\mathrm{T} 1 \mathrm{C}$ & $46 \pm 30$ \\
\hline FLAIR & $84 \pm 44$ \\
\hline Isotropic (p) DTI & $83 \pm 44$ \\
\hline Anisotropic (q) DTI & $51 \pm 23$ \\
\hline \multicolumn{2}{|l|}{ EOR, by imaging type, $\%$} \\
\hline Postcontrast T1-weighted image & $136 \pm 71$ \\
\hline FLAIR & $58 \pm 21$ \\
\hline Isotropic (p) DTI & $57 \pm 18$ \\
\hline Anisotropic (q) DTI & $83 \pm 20$ \\
\hline \multicolumn{2}{|l|}{ Residual tumor size, by imaging type, $\mathrm{ml}$} \\
\hline Postcontrast T1-weighted image & $2.7 \pm 6.7$ \\
\hline FLAIR & $41 \pm 32$ \\
\hline Isotropic (p) DTI & $38 \pm 30$ \\
\hline Anisotropic (q) DTI & $8 \pm 9.6$ \\
\hline GTR, $†$ no. of patients & 24 \\
\hline STR, $\dagger$ no. of patients & 7 \\
\hline IDH-1 positive, no. of patients & 3 \\
\hline MGMT methylation positive, no. of patients & 10 \\
\hline Progression-free survival, days & $367 \pm 263$ \\
\hline Overall survival, days & $559 \pm 292$ \\
\hline
\end{tabular}

GTR = gross-total resection; STR = subtotal resection; T1C = postcontrast T1-weighted image.

* Data are given as mean \pm SD unless otherwise indicated.

$\dagger$ Based on contrast-enhanced T1-weighted image.

$\mathrm{ml}$; range $10-131 \mathrm{ml}$ ), which was significantly larger than the presurgical tumor $(p=0.001)$. A total of $57 \%$ of the abnormal $\mathrm{p}$ area, $83 \%$ of the abnormal $\mathrm{q}$ area, and $59 \%$ of the increased FLAIR signal area was resected. Residual tumor volume based on p, q, FLAIR, and postcontrast T1weighted images was $38.4 \mathrm{ml}( \pm 30.2 \mathrm{ml}$; range 4.4-129.4 $\mathrm{ml}), 8 \mathrm{ml}( \pm 9.7 \mathrm{ml}$; range $0-36 \mathrm{ml}), 40.7 \mathrm{ml}( \pm 32.7 \mathrm{ml}$; range $0.4-127.9 \mathrm{ml})$, and $2.7 \mathrm{ml}( \pm 6.8 \mathrm{ml}$; range $0-26.6$ $\mathrm{ml}$ ), respectively. None of the patients had a major postsurgical neurological deficit or Karnofsky Performance Scale score of less than 70, which was our condition to undergo temozolomide chemoradiotherapy.

\section{EOR and Patient Outcome}

Univariate Cox regression models for each variable showed a significant correlation of progression-free survival with the EOR of the $\mathrm{p}$ area $(\mathrm{p}=0.030)$, complete resection of the contrast-enhanced lesion $(p=0.004)$, and MGMT methylation status $(\mathrm{p}=0.041)$. Multivariate analysis was used to test the EOR with other covariates (Table 2 ). The results showed that resection of the more abnormal $\mathrm{p}$ was a protective predictor of tumor progression (HR $0.911 ; p=0.009)$. The EOR of abnormal $q$ areas was also significantly correlated with progression-free survival in the multivariate analysis (HR 0.935; $\mathrm{p}=0.006$ ). The EOR based on FLAIR showed no association with progressionfree survival in either univariate or multivariate analysis ( $p=0.994$ and $p=0.799$, respectively). The presence of MGMT methylation was found to be a significant predictor of progression-free survival in the multivariate models for $\mathrm{p}$ (HR 4.626; $\mathrm{p}=0.009)$, q (HR 6.716; $\mathrm{p}=0.006)$, and FLAIR (HR 95.941; $\mathrm{p}=0.001$ ).

For overall survival, multivariate analysis was performed after controlling for age, MGMT methylation status, IDH-1 mutation status, presurgical tumor volume, tumor eloquence, and midline shift. Both the EOR based on the q map and the EOR based on postcontrast T1-weighted images were identified as predictors for overall survival (HR 0.965, p = 0.041; HR 9.946, $\mathrm{p}=0.050$, respectively). The MGMT methylation status was significantly associated with overall survival in the multivariate models for $\mathrm{p}$ map (HR 3.737; $\mathrm{p}=0.043$ ), q map (HR 4.932; $\mathrm{p}=0.012$ ), and FLAIR (HR 10.274; $p=0.009$ ) images. Presurgical tumor volume based on postcontrast T1-weighted images was found to be a covariate associated with increased hazard ratio relative to EOR of the q abnormality (HR 1.039; $p=0.024)$ and on the enhanced area of the postcontrast T1-weighted image (HR 1.037; $\mathrm{p}=0.040)$.

Previous results indicated the importance of the EOR, especially q abnormality, on outcome; therefore, we explored this in more detail. Classifying patients into 2 groups by using the median of the extent of abnormal $\mathrm{q}$ resection resulted in a resection cutoff of $89 \%$ of the $\mathrm{q}$ abnormality. Patients with a resection of greater than $89 \%$ of the q abnormality had a significantly longer progression-free survival (mean $421 \pm 311$ days) than those with a resection of less than $89 \%$ of the abnormality $(257 \pm 214$ days; $\mathrm{p}=0.034)$ and better overall survival $(621.9 \pm 389.0$ days vs $518.13 \pm 264.7$ days; $p=0.011$ ) (Fig. 2). There was no statistical difference between these 2 subgroups in age, sex, tumor location, number of patients receiving a grosstotal resection, or MGMT methylation or IDH-1 mutation status (Table 3). Similar results were seen when subgrouping patients with the median extent of $\mathrm{p}$ resection: Longer progression-free survival was shown in patients with greater than $60 \%$ resection of the p abnormal area ( $421 \pm$ 311 vs $258 \pm 176 ; \mathrm{p}=0.046$ ).

In our study, 26 patients (83.9\%) had tumor recurrence within $2 \mathrm{~cm}$ adjacent to the resection cavity. Three patients (9.7\%) had distal recurrence more than $2 \mathrm{~cm}$ from the original resection cavity, and 2 had recurrence both locally and distally. All patients with solely distal recurrence received complete resection of the enhanced lesion shown on the postcontrast T1-weighted image and a greater EOR of the q abnormal area (97.7\%) than the others (87.4\%), and 2 were MGMT methylated. Progression-free survival (721 \pm 270 days) and overall survival (954 \pm 461 days) were also longer in patients with distal recurrence. 
TABLE 2. Extent of resection and patient outcome*

\begin{tabular}{ccccccccc}
\hline & \multicolumn{3}{c}{ Progression-Free Survival } & & \multicolumn{3}{c}{ Overall Survival } \\
\cline { 2 - 3 } \cline { 7 - 8 } EOR, by Imaging Type & $p$ Value & HR & $95 \% \mathrm{Cl}$ & & $p$ Value & HR & $95 \% \mathrm{Cl}$ \\
\hline$p$ & $0.009 \dagger$ & 0.911 & $0.850-0.977$ & & 0.795 & 0.993 & $0.940-1.049$ \\
\hline$q$ & $0.006 \dagger$ & 0.935 & $0.891-0.980$ & & $0.041 \dagger$ & 0.965 & $0.934-0.999$ \\
\hline FLAIR & 0.799 & 0.997 & $0.926-1.061$ & & 0.052 & 1.062 & $1.999-1.129$ \\
\hline T1C & 0.094 & 6.499 & $0.727-58.069$ & & $0.050 \dagger$ & 9.946 & $1.005-98.464$ \\
\hline
\end{tabular}

* Multivariate analysis results showing age, MGMT methylation status, IDH-1 mutation status, presurgical tumor volume based on postcontrast

T1-weighted, midline shift, and tumor eloquent location as covariates.

$\dagger$ Statistically significant.

\section{Residual Tumor Volume and Patient Outcome}

The correlations of patients' outcome and residual tumor volume based on different MR images are summarized in Table 4. A larger residual volume on DTI was associated with a decrease in progression-free survival, which was statistically significant for the $\mathrm{q}$ abnormality (HR 1.118; $\mathrm{p}=0.008$ ), while residual volume of the $\mathrm{p}$ abnormality was not significant (HR 1.28; $\mathrm{p}=0.074$ ). Residual FLAIR volume was not correlated with progression-free survival. Overall survival was not influenced by the residual abnormal p, q, or contrast-enhanced lesion volumes. Residual FLAIR volume decreased the hazard ratio of overall survival (HR 0.942; $p=0.008$ ).

\section{Discussion}

In this study, we retrospectively reviewed the correlation between the EOR based on DTI and patients' outcome. Although the intention of initial resection was

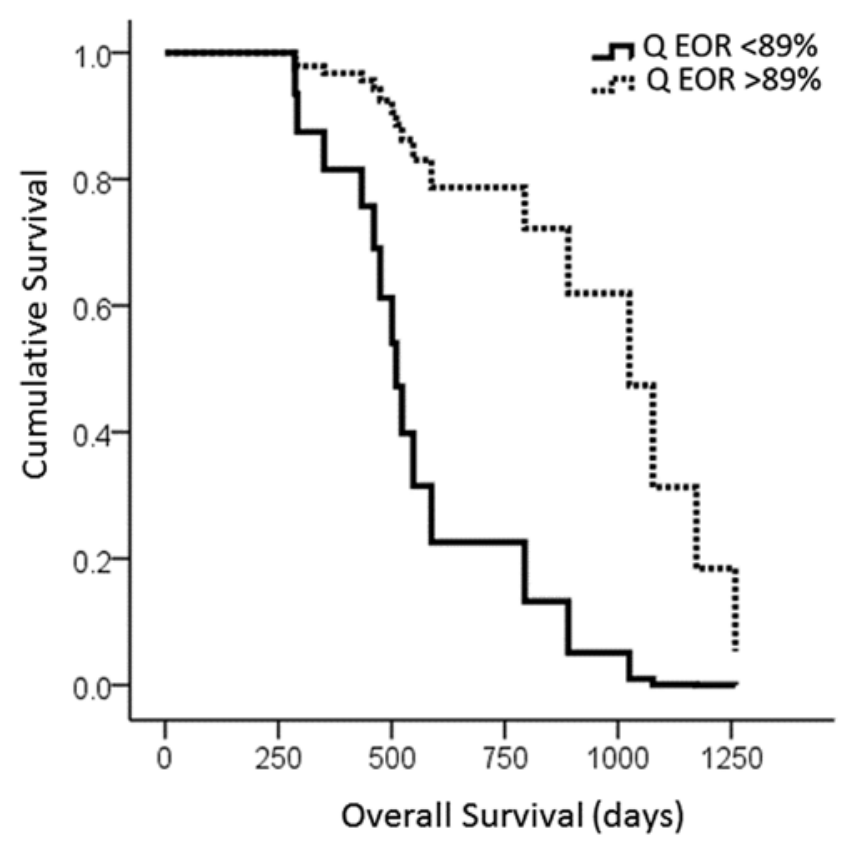

FIG. 2. Patient overall survival according to abnormal q resection ratio. Cox regression survival analysis showed that patients with a resection of greater than $89 \%$ of the $q$ abnormality had a better overall survival than patients with less than $89 \%$ resection $(p=0.011)$. based on 5-ALA rather than on any DTI parameter, this study showed, via multivariate Cox regression model, a significant correlation between the EOR, based on both the $\mathrm{p}$ map and q map, and progression-free survival. Furthermore, a favorable overall survival was seen in patients who received greater resection of the q map and the enhanced area on postcontrast T1-weighted images. Thus, by resecting more abnormal DTI areas, most importantly the $\mathrm{q}$ area, the infiltrating tumor burden decreases, leading to a better outcome.

The significance of resecting more of a contrast-enhanced lesion has been shown clearly to correlate with patient survival. Sanai and colleagues showed that improved overall survival begins at a $78 \%$ resection and continues to increase as resection of the contrast-enhanced area becomes greater. ${ }^{21}$ A nonvolumetric study of high-grade glioma has also shown a longer overall survival associated with complete resection rather than with incomplete resection (16.7 vs 11.8 months, respectively; $p<0.001){ }^{25}$ Others have reported the synergic clinical benefit of the EOR and concomitant chemoradiotherapy. ${ }^{23}$ Moreover, the benefit of reduced tumor burden is related to the efficacy of 1,3-bis(2-chloroethyl)-1-nitrosourea (BCNU): In one study, a lower concentration was needed to achieve $90 \%$ growth inhibition in low-tumor-burden groups. ${ }^{14} \mathrm{In}$ patients receiving $\mathrm{BCNU}$ wafers during surgery, longer median survival has been noted in the complete-resection group compared with those in the subtotal-resection group. ${ }^{26}$ Therefore, reduction of contrast-enhancing tumor burden is an important prognostic factor for patient outcome.

To date, most studies have defined EOR using the contrast-enhanced area only; however, a false-negative rate of $16 \%$ was found in normal-appearing areas on T1weighted images ${ }^{17}$ and tumor cells often extend beyond the contrast-enhanced area. We have shown previously that specific DTI signatures can predict this microscopic tumor invasion..$^{17,18}$ In particular, regions with greater than a $10 \%$ increase of $p$ signified white matter infiltration by tumor, whereas regions with greater than a $12 \%$ decrease in q showed white matter disruption by cancer. Therefore, using DTI can better delineate the actual tumor margin and show the invasive area of tumor. Although we did not perform a histological correlation in this study, previous research has validated a correlation between DTI and viable tumor cells. ${ }^{4}$

A previously conducted tumor-resection treatment- 
TABLE 3. Patient characteristics for the subgroups of q resection area*

\begin{tabular}{|c|c|c|c|}
\hline Characteristic & q EOR $<89 \%$ & q EOR $>89 \%$ & $\mathrm{p}$ Value \\
\hline Male & 9 & 10 & 1 \\
\hline Female & 6 & 6 & \\
\hline Age, yrs & $53.67 \pm 13.16$ & $58.68 \pm 8.8$ & 0.4168 \\
\hline Tumor location, no. & & & 0.1298 \\
\hline Eloquent & 5 & 5 & \\
\hline Near eloquent & 8 & 4 & \\
\hline Noneloquent & 2 & 7 & \\
\hline Midline shift, mm & $3.33 \pm 3.90$ & $3.65 \pm 3.63$ & 0.8138 \\
\hline \multicolumn{4}{|l|}{ Presurgical tumor size, by imaging type, $\mathrm{ml}$} \\
\hline $\mathrm{T} 1 \mathrm{C}$ & $35.9 \pm 18.4$ & $53.3 \pm 30.6$ & 0.0682 \\
\hline FLAIR & $81.1 \pm 37.8$ & $95.8 \pm 52.2$ & 0.3888 \\
\hline Isotropic (p) DTI & $80.1 \pm 38.1$ & $90.9 \pm 48.2$ & 0.4948 \\
\hline Anisotropic (q) DTI & $48.8 \pm 14.3$ & $54.5 \pm 30.8$ & 0.5147 \\
\hline GTR, $\ddagger$ no. of patients & 9 & 15 & 0.0693 \\
\hline STR, $\ddagger$ no. of patients & 6 & 1 & \\
\hline IDH-1 positive, no. of patients & 2 & 1 & 0.5050 \\
\hline MGMT methylation positive, no. of patients & 5 & 5 & 0.9013 \\
\hline Progression-free survivalł, days & $257 \pm 214$ & $421 \pm 311$ & $0.034 \dagger$ \\
\hline Overall survival, $\S$ days & $518.13 \pm 264.7$ & $621.9 \pm 389.0$ & $0.011 \dagger$ \\
\hline
\end{tabular}

bias study showed that complete resection was more often achieved in younger patients and in those with tumors in noneloquent tumor locations. ${ }^{25}$ We tested our result by using a median q-abnormality EOR of $89 \%$ as the cutoff to stratify patients into 2 groups. In these groups, a longer progression-free survival was seen in those with a greater than $89 \%$ q-abnormality resection, but other variables, including age, tumor eloquence, MGMT methylation status, IDH mutation, midline shift, presurgical tumor size, and complete resection of the enhanced lesion on postcontrast T1-weighted images, were all comparable (Table 3). A bias, therefore, could not be identified, which strengthens our results, indicating the importance of the EOR based on DTI.

Specifically looking at the patients with distal recurrence, a previous study showed a correlation between extended resection and recurrence pattern, with a better prognosis in those with distal recurrence. ${ }^{3}$ In our study, all
3 patients with distal recurrence received a greater EOR of the DTI-detected abnormality (EOR of q abnormality $>97 \%$ ) beyond the contrast-enhanced area. Although the number in our study is small, this finding may indicate that distal recurrence occurs in those with a better local control based on DTI and consequently resulted in a better prognosis.

We also examined the residual tumor volume based on different MR sequences. More abnormal q volume left after surgery can significantly increase the risk of progression and marginally decrease overall survival. Grabowski and colleagues showed that residual contrast-enhanced lesion volume of more than $2 \mathrm{~cm}^{3}$ after surgical resection was a strong, unfavorable predictor of overall survival. ${ }^{5}$ Furthermore, others also concluded that a residual contrast-enhanced tumor volume of less than $10 \mathrm{~cm}^{3}$ can lead to both prolonged time to progression and survival. ${ }^{11}$ In our results, only marginal significance in progression-free

TABLE 4. Residual tumor volume and patient outcome*

\begin{tabular}{llllllll}
\hline & \multicolumn{3}{c}{ Progression-Free Survival } & & \multicolumn{3}{c}{ Overall Survival } \\
\cline { 2 - 3 } \cline { 7 - 8 } Residual Tumor & $\mathrm{p}$ Value & $\mathrm{HR}$ & $95 \% \mathrm{Cl}$ & & $\mathrm{p}$ Value & $\mathrm{HR}$ & $95 \% \mathrm{Cl}$ \\
\hline T1C & 0.060 & 1.393 & $0.986-1.969$ & & 0.401 & 1.140 & $0.840-1.549$ \\
\hline Isotropic (p) DTI & 0.074 & 1.028 & $0.997-1.059$ & & 0.942 & 0.999 & $0.970-1.029$ \\
\hline Anisotropic (q) DTI & $0.008 \dagger$ & 1.118 & $1.029-1.215$ & & 0.080 & 1.053 & $0.994-1.116$ \\
\hline FLAIR & 0.882 & 1.003 & $0.968-1.038$ & & $0.008 \dagger$ & 0.939 & $0.897-0.983$ \\
\hline
\end{tabular}

* Multivariate analysis results showing age, MGMT methylation status, IDH-1 mutation status, presurgical tumor volume based on T1 contrast imaging, midline shift, and tumor eloquent location as covariates.

† Statistically significant. 
survival was noted based on postcontrast T1-weighted imaging. This may be due to the limited numbers of patients in our study. Regardless of the limited numbers, we clearly displayed the advantage of the q map, which, according to a previous biopsy studies, represents regions of tumor cells ${ }^{17}$ Thus, a smaller residual abnormality in the q region indicated a lower tumor load and a better prognosis.

\section{Conclusions}

The expanding application of DTI in patients with brain tumors can demonstrate not only possible tumor invasion but also can provide a guide for surgeons. Our results underscore the importance of abnormal DTI area, especially of abnormal q area, showing that patients who received larger EOR and who had less residual abnormal DTI had better progression-free survival and overall survival. Further prospective studies are needed to clarify the clinical benefit of incorporating DTI into surgical planning.

\section{Acknowledgments}

This research was funded by the UK National Institute of Health Research Clinician Scientist Fellowship (S.J.P.) and grants from Chang Gung Medical Foundation and Chang Gung Memorial Hospital (J.L.Y.), the Remmert Adriaan Laan Fund (A.H.), the René Vogels Fund (A.H.), and the Commonwealth Scholarship Commission and Cambridge Commonwealth Overseas Trust (N.R.B.).

\section{References}

1. Berberat J, McNamara J, Remonda L, Bodis S, Rogers S: Diffusion tensor imaging for target volume definition in glioblastoma multiforme. Strahlenther Onkol 190:939-943, 2014

2. Castellano A, Donativi M, Ruda R, De Nunzio G, Riva M, Iadanza A, et al: Evaluation of low-grade glioma structural changes after chemotherapy using DTI-based histogram analysis and functional diffusion maps. Eur Radiol [epub ahead of print], 2015

3. De Bonis P, Anile C, Pompucci A, Fiorentino A, Balducci $\mathrm{M}$, Chiesa S, et al: The influence of surgery on recurrence pattern of glioblastoma. Clin Neurol Neurosurg 115:37-43, 2013

4. Ellingson BM, Malkin MG, Rand SD, Connelly JM, Quinsey C, LaViolette PS, et al: Validation of functional diffusion maps (fDMs) as a biomarker for human glioma cellularity. J Magn Reson Imaging 31:538-548, 2010

5. Grabowski MM, Recinos PF, Nowacki AS, Schroeder JL, Angelov L, Barnett GH, et al: Residual tumor volume versus extent of resection: predictors of survival after surgery for glioblastoma. J Neurosurg 121:1115-1123, 2014

6. Hardesty DA, Sanai N: The value of glioma extent of resection in the modern neurosurgical era. Front Neurol 3:140, 2012

7. Hentschel SJ, Sawaya R: Optimizing outcomes with maximal surgical resection of malignant gliomas. Cancer Contr 10:109-114, 2003

8. Jena R, Price SJ, Baker C, Jefferies SJ, Pickard JD, Gillard $\mathrm{JH}$, et al: Diffusion tensor imaging: possible implications for radiotherapy treatment planning of patients with high-grade glioma. Clin Oncol (R Coll Radiol) 17:581-590, 2005

9. Jenkinson M, Bannister P, Brady M, Smith S: Improved optimization for the robust and accurate linear registration and motion correction of brain images. Neuroimage 17:825-841, 2002

10. Jenkinson M, Smith S: A global optimisation method for robust affine registration of brain images. Med Image Anal 5:143-156, 2001

11. Keles GE, Lamborn KR, Chang SM, Prados MD, Berger MS: Volume of residual disease as a predictor of outcome in adult patients with recurrent supratentorial glioblastomas multiforme who are undergoing chemotherapy. J Neurosurg 100:41-46, 2004

12. Lacroix M, Abi-Said D, Fourney DR, Gokaslan ZL, Shi W, DeMonte F, et al: A multivariate analysis of 416 patients with glioblastoma multiforme: prognosis, extent of resection, and survival. J Neurosurg 95:190-198, 2001

13. Li YM, Suki D, Hess K, Sawaya R: The influence of maximum safe resection of glioblastoma on survival in 1229 patients: Can we do better than gross-total resection? J Neurosurg [Epub ahead of print October 23, 2015. DOI: 10.3171/2015.5.JNS142087]

14. Ng WH, Wan GQ, Too HP: Higher glioblastoma tumour burden reduces efficacy of chemotherapeutic agents: in vitro evidence. J Clin Neurosci 14:261-266, 2007

15. Ostrom QT, Gittleman H, Liao P, Rouse C, Chen Y, Dowling J, et al: CBTRUS statistical report: primary brain and central nervous system tumors diagnosed in the United States in 2007-2011. Neuro Oncol 16 (Suppl 4):iv1-iv63, 2014

16. Peña A, Green HA, Carpenter TA, Price SJ, Pickard JD, Gillard JH: Enhanced visualization and quantification of magnetic resonance diffusion tensor imaging using the $\mathrm{p}: \mathrm{q}$ tensor decomposition. Br J Radiol 79:101-109, 2006

17. Price SJ, Jena R, Hutchinson PJ, Dean AF, Peña A Pickard $\mathrm{JD}$, et al: Improved delineation of glioma margins and regions of infiltration with use of diffusion tensor imaging: an image-guided biopsy study. AJNR Am J Neuroradiol 27:1969-1974, 2006

18. Price SJ, Peña A, Burnet NG, Jena R, Green HA, Carpenter TA, et al: Tissue signature characterisation of diffusion tensor abnormalities in cerebral gliomas. Eur Radiol 14:19091917, 2004

19. Price SJ, Waldman AD: Advances in imaging brain cancer, in Watts C (ed): Emerging Concepts in Neuro-Oncology. London: Springer, 2013, pp 119-140

20. Sahm F, Capper D, Jeibmann A, Habel A, Paulus W, Troost $\mathrm{D}$, et al: Addressing diffuse glioma as a systemic brain disease with single-cell analysis. Arch Neurol 69:523-526, 2012

21. Sanai N, Polley MY, McDermott MW, Parsa AT, Berger MS: An extent of resection threshold for newly diagnosed glioblastomas. J Neurosurg 115:3-8, 2011

22. Sawaya R, Hammoud M, Schoppa D, Hess KR, Wu SZ, Shi WM, et al: Neurosurgical outcomes in a modern series of 400 craniotomies for treatment of parenchymal tumors. Neurosurgery 42:1044-1056, 1998

23. Stummer W, Meinel T, Ewelt C, Martus P, Jakobs O, Felsberg J, et al: Prospective cohort study of radiotherapy with concomitant and adjuvant temozolomide chemotherapy for glioblastoma patients with no or minimal residual enhancing tumor load after surgery. J Neurooncol 108:89-97, 2012

24. Stummer W, Pichlmeier U, Meinel T, Wiestler OD, Zanella F, Reulen HJ: Fluorescence-guided surgery with 5-aminolevulinic acid for resection of malignant glioma: a randomised controlled multicentre phase III trial. Lancet Oncol 7:392401, 2006

25. Stummer W, Reulen HJ, Meinel T, Pichlmeier U, Schumacher W, Tonn JC, et al: Extent of resection and survival in glioblastoma multiforme: identification of and adjustment for bias. Neurosurgery 62:564-576, 2008

26. Stummer W, van den Bent MJ, Westphal M: Cytoreductive surgery of glioblastoma as the key to successful adjuvant therapies: new arguments in an old discussion. Acta Neurochir (Wien) 153:1211-1218, 2011

27. Stupp R, Hegi ME, Mason WP, van den Bent MJ, Taphoorn 
MJ, Janzer RC, et al: Effects of radiotherapy with concomitant and adjuvant temozolomide versus radiotherapy alone on survival in glioblastoma in a randomised phase III study: 5-year analysis of the EORTC-NCIC trial. Lancet Oncol 10:459-466, 2009

\section{Disclosures}

The authors report no conflict of interest concerning the materials or methods used in this study or the findings specified in this paper. This paper presents independent research funded by the NIHR. The views expressed are those of the author(s) and not necessarily those of the UK National Health Service, the NIHR, or the UK Department of Health.

\section{Author Contributions}

Conception and design: Yan, van der Hoorn, Price. Acquisition of data: Yan, van der Hoorn, Matys. Analysis and interpretation of data: Yan, van der Hoorn. Drafting the article: Yan, van der Hoorn, Larkin, Boonzaier, Price. Critically revising the article: all authors. Reviewed submitted version of manuscript: Yan. Approved the final version of the manuscript on behalf of all authors: Yan. Statistical analysis: Yan. Administrative/technical/ material support: Yan, van der Hoorn, Larkin, Boonzaier. Study supervision: Price.

\section{Correspondence}

Jiun-Lin Yan, Department of Clinical Neuroscience, University of Cambridge, Addenbrooke's Hospital, Box 167, Cambridge CB2 0QQ, United Kingdom. email: jly27@cam.ac.uk. 